\title{
CASCIRE surveillance network and work on avian influenza viruses
}

Yuhai $\mathrm{Bi}^{1,2^{*}}$, Weifeng $\mathrm{Shi}^{3}$, Jianjun $\mathrm{Chen}^{4}$, Quanjiao $\mathrm{Chen}^{4}$, Zhenghai $\mathrm{Ma}^{5}$, Gary Wong ${ }^{2}$, Wenxia Tian ${ }^{6}$, Renfu Yin ${ }^{7}$, Guanghua Fu ${ }^{8}$, Yongchun Yang, William J. Liu ${ }^{10}$, Chuansong Quan ${ }^{10}$, Qianli Wang ${ }^{11}$, Shenghu $\mathrm{He}^{12}$, Xiangdong $\mathrm{Li}^{13}$, Qianfeng Xia ${ }^{14}$, Lixin Wang ${ }^{14}$, Zhaohui $\mathrm{Pan}^{15}$, Laixing $\mathrm{Li}^{16}$, Hong $\mathrm{Li}^{17}$, Wen $\mathrm{Xu}^{17}$, Ying Luo ${ }^{18}$, Hui Zeng ${ }^{19}$, Lianpan Dai $^{20}$, Haixia Xiao ${ }^{21}$, Kirill Sharshov ${ }^{22}$, Alexander Shestopalov ${ }^{22}$, Yi Shi ${ }^{1,2}$, Jinghua Yan ${ }^{1,2}$, Xuebing $\mathrm{Li}^{1}$, Yingxia Liu ${ }^{2}$, Fumin Lei $^{20}$, Wenjun Liu ${ }^{1}$ \& George F. Gao ${ }^{1,2,10^{*}}$

${ }^{1}$ CAS Key Laboratory of Pathogenic Microbiology and Immunology, Collaborative Innovation Center for Diagnosis and Treatment of Infectious Disease, Institute of Microbiology, Center for Influenza Research and Early-warning (CASCIRE), Chinese Academy of Sciences, Beijing 100101, China;

${ }^{2}$ Shenzhen Key Laboratory of Pathogen and Immunity, State Key Discipline of Infectious Disease, Shenzhen Third People's Hospital, Shenzhen 518112, China;

${ }^{3}$ Shandong Universities Key Laboratory of Etiology and Epidemiology of Emerging Infectious Diseases, Taishan Medical College, Tai'an 271016, China;

${ }^{4}$ CAS Key Laboratory of Special Pathogens and Biosafety, Wuhan Institute of Virology, Chinese Academy of Sciences, Wuhan 430071, China; ${ }^{5}$ College of Life Science and Technology, Xinjiang University, Urumchi 830046, China;

${ }^{6}$ College of Animal Science and Veterinary Medicine, Shanxi Agricultural University, Taigu 030801, China;

${ }^{7}$ Department of Veterinary Preventive Medicine, College of Veterinary Medicine, Jilin University, Jilin 130062, China;

${ }^{8}$ Institute of Animal Husbandry and Veterinary Medicine, Fujian Academy of Agricultural Sciences, Fuzhou 350013, China;

${ }^{9}$ Center of Excellence for Animal Health Inspection, College of Animal Science and Technology, Zhejiang Agriculture and Forestry University, Zhejiang 311300, China;

${ }^{10}$ National Institute for Viral Disease Control and Prevention, Chinese Center for Disease Control and Prevention (China CDC), Beijing 102206, China;

${ }^{11}$ Key Laboratory of Public Health Safety, Ministry of Education, School of Public Health, Fudan University, Shanghai 200032, China ;

${ }^{12}$ Laboratory of Clinical Veterinary Medicine, College of Agriculture, Ningxia University, Yinchuan 750021, China;

${ }^{13}$ National Research Center for Veterinary Medicine, Luoyang 471003, China;

${ }^{14}$ Laboratory of Tropical Biomedicine and Biotechnology, and Faculty of Tropical Medicine and Laboratory Medicine, Immunology

Department, School of Basic Medicine and Life Science, Hainan Medical University, Haikou 571101, China;

${ }^{15}$ Xizang Agriculture and Animal Husbandry College, Linzhi 860000, China;

${ }^{16}$ Key Laboratory of Adaptation and Evolution of Plateau Biota, Northwest Institute of Plateau Biology, Chinese Academy of Sciences, Xining 810000, China;

${ }^{17}$ Yunnan Center for Disease Control and Prevention, Kunming 650022, China;

${ }^{18}$ Department of Wildlife Conservation and Nature Reserve Management, State Forestry Administration, Beijing 100714, China;

${ }^{19}$ Institute of Infectious Diseases, Beijing Ditan Hospital, Capital Medical University, Beijing 100015, China;

${ }^{20}$ Institute of Zoology, Chinese Academy of Sciences, Beijing 100101, China;

${ }^{21}$ Laboratory of Protein Engineering and Vaccines, Tianjin Institute of Industrial Biotechnology, Chinese Academy of Sciences, Tianjin 300308, China;

${ }^{22}$ Research Institute of Experimental and Clinical Medicine, Novosibirsk State University, Novosibirsk 630090, Russia

Received November 16, 2017; accepted November 23, 2017; published online December 1, 2017

\footnotetext{
*Corresponding authors (Yuhai Bi, email: beeyh@im.ac.cn; George F. Gao, email: gaof@im.ac.cn)
} 
Citation: Bi, Y., Shi, W., Chen, J., Chen, Q., Ma, Z., Wong, G., Tian, W., Yin, R., Fu, G., Yang, Y., Liu, W.J., Quan, C., Wang, Q., He, S., Li, X., Xia, Q., Wang, L., Pan, Z., Li, L., Li, H., Xu, W., Luo, Y., Zeng, H., Dai, L., Xiao, H., Sharshov, K., Shestopalov, A., Shi, Y., Yan, J., Li, X., Liu, Y., Lei, F., Liu, W., and Gao, G.F. (2017). CASCIRE surveillance network and work on avian influenza viruses. Sci China Life Sci 60, 1386-1391. https://doi.org/ $10.1007 / \mathrm{s} 11427-017-9251-2$

Studies on influenza virus by Chinese Academy of Sciences (CAS) could be traced back as early as 2005 by the CAS Key Laboratory of Pathogenic Microbiology and Immunology (CASPMI), who discovered that Qinghai-like Clade 2.2 H5N1 subtype highly pathogenic avian influenza virus (HPAIV) first caused severe outbreak in wild birds in Qinghai Lake (Liu et al., 2005).

Since then, to set the platform for further investigative work, CASPMI has worked continuously on the surveillance, genetic evolution, pathogenesis, cross-species infections to mammals and humans, antivirals, antibodies and vaccines against influenza virus, as well as other emerging infectious pathogens. In 2014, the CAS Center for Influenza Research and Early-warning (CASCIRE) (http://www.im. cas.cn/xwzx/jqyw/201412/t20141229_4283087.html) and the Network Surveillance Unit (NUS) of CASCIRE (Figure 1), as well as the joint-lab between CASCIRE and the regions of The Belt and Road (e.g. Russia) have been developing a coordinated emergency response and research capacity on emerging or re-emerging infectious diseases. In this article, the aims of CASCIRE and its work on influenza were summarized, with the aim of promoting collaborations between CASCIRE and other research groups for better prevention and control of emerging or re-emerging infectious diseases.

\section{THE ROLES OF MIGRATORY BIRDS IN THE EVOLUTION AND TRANSMISSION OF HPAIVS}

During our surveillance studies, the Qinghai-like Clade 2.2 H5N1 virus was identified again in 2006 at Qinghai Lake. While the virus possessed some differences in its genome compared to those isolated in 2005, and was more similar to those identified in Asia, Europe and Africa along the migratory flyways of wild birds. We then hypothesized that wild birds play important roles for the spread, transmission and evolution of HPAIVs worldwide through their migratory activities (Wang et al., 2008). Currently, the Qinghai-like Clade 2.2 H5N1 virus has evolved into different sub-clades in poultry, is dominant and occasionally causes sporadic human infections in Egypt (http://www.who.int/influenza/ vaccines/virus/characteristics_virus_vaccines/en/).

Our hypothesis was further supported by the novel reassortant SMX-like Clade 2.3.2.1c H5N1 virus, which evolved from Clade 2.3.2 found in 2009 (Hu et al., 2011), in whooper swans and wild ducks in Sanmenxia city of the
Yellow River Region in 2015 (Bi et al., 2015d). The biological characteristics, including drug sensitivity screening and pathogenicity in chickens and mice were studied in our laboratory, and three diseased whooper swans were treated with sensitive drugs and cured. Moreover, CASCIRE was able to warn about the spread of the SMX-like Clade 2.3.2.1c $\mathrm{H} 5 \mathrm{~N} 1$ based on the flyways of wild birds. Based on this early warning, the SMX-like viruses were quickly identified and treated in wild birds in Inner Mongolia and Qinghai Lake (Bi et al., 2016a) (http://www.im.cas.cn/xwzx/jqyw/201509/ t20150902_4419497.html). Due to the typical genetic characteristics with Clade 2.3.2.1c HA and H9N2-derived PB2 gene, the SMX-like viruses were easy to be differentiated and were again identified in wild birds and poultry in other Asian and European regions along the flyways during 20142015. The viruses were found to possess mutations in its genome, indicating viral evolution (Bi et al., 2016a). Studies on SMX-like H5N1 virus further supported our viewpoints on the roles of migratory birds in the evolution and transmission of HPAIVs. As a result, our hypothesis proposing that HPAI spread is facilitated over long distances by migratory wild birds is now largely accepted by the scientific community, especially after the worldwide transmission of H5N8 HPAIVs (http://www.oie.int/en/animal-health-in-theworld/update-on-avian-influenza).

\section{THE ROLES OF LIVE POULTRY MARKETS IN THE EVOLUTION AND TRANSMISSION OF NOVEL AIVS}

The CASCIRE surveillance network monitors wildlife (e.g. wild birds), domestic animals (e.g. poultry), and includes sentinel hospitals for human cases, forming a complete circle for monitoring novel pathogens that pose potential risks to humans and animals alike. Human AIV infections may occasionally occur after exposure to the virus from live poultry or the environment, e.g. live poultry markets (LPMs) (http:// www.who.int/influenza/human_animal_interface/HAI_Risk_Assessment/en/). A majority of viruses isolated from human cases possessed high genetic similarity to viruses from LPMs, such as H10N8 and H5N6 (Bi et al., 2015a; Bi et al., 2016b; Zhang et al., 2014). In addition, the novel AIVs, such as H7N9 and H5N6, are evolving, spreading and undergoing dynamic reassortment with low pathogenicity avian influenza viruses (LPAIVs) (e.g. H9N2) in LPMs (Bi et al., 2016b; Cui et al., 2014). Therefore, the LPMs were considered as "incubators" for the evolution and emergence of 


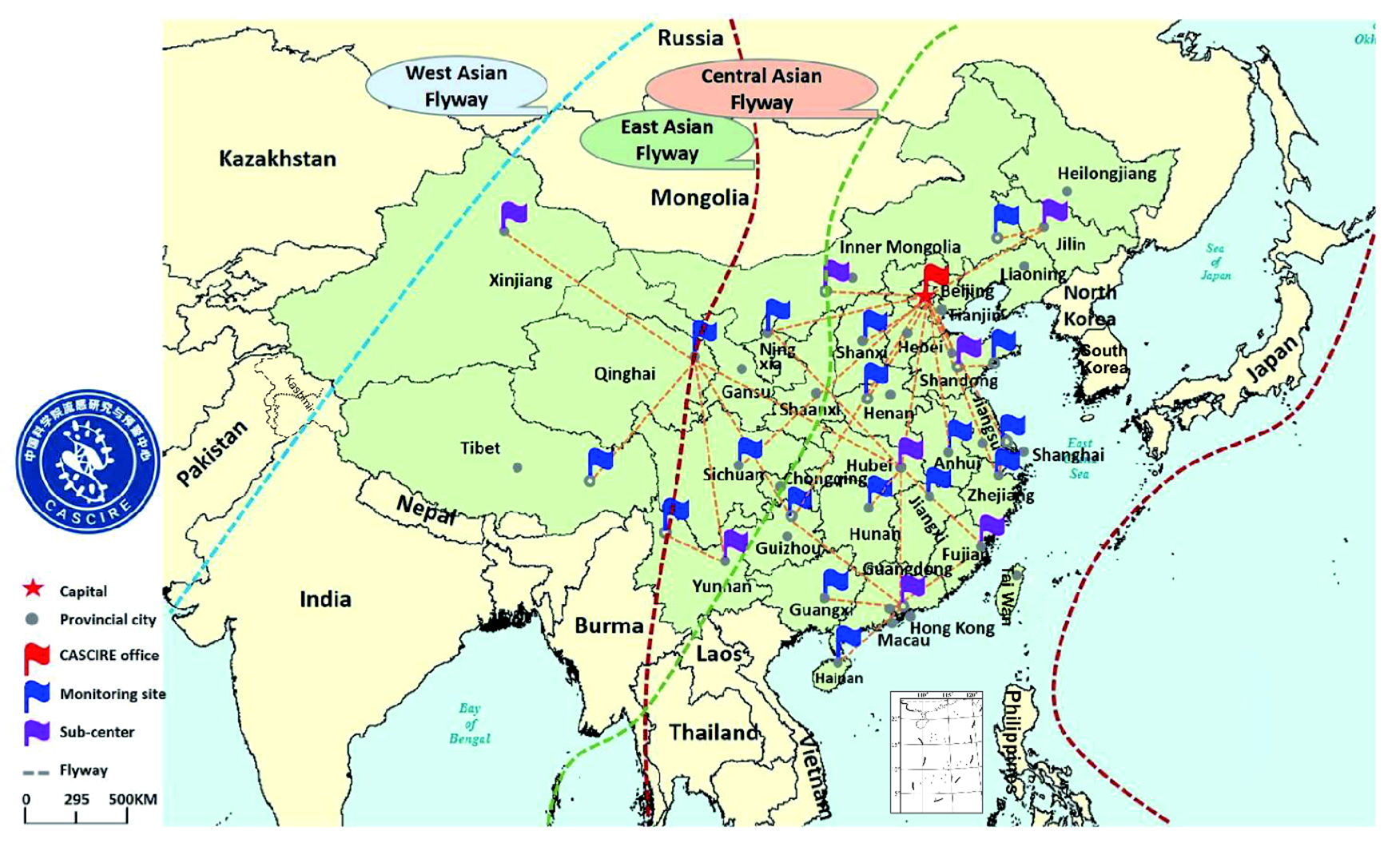

Figure 1 The surveillance network of CASCIRE.

novel AIVs (Gao, 2014). Interestingly, the internal genes of novel, dominant H7N9, H10N8, and H5N6 AIVs were all found to originate from H9N2 LPAIVs (Bi et al., 2016b; Liu et al., 2013; Zhang et al., 2014). Novel AIVs carried by wild birds could be transmitted to domestic birds through direct or indirect contact, and then the HA and NA genes of the novel viruses were conserved and adapted to poultry by rapid reassortment with the internal genes of a poultry-adapted AIVs (e.g. H9N2), to help novel reassortants replicate and evolve in domestic poultry ( $\mathrm{Su}$ et al., 2015). Thus, the poultryadapted H9N2 may increase adaptation of aquatic bird origin HA and NA genes to domestic birds (Liu et al., 2014).

We also discovered that the H7N9 HPAIV variant in poultry from LPMs as early as July 2016 (Qi et al., 2017), and have subsequently identified several human cases (Zhang et al., 2017). Notably, we also found that H5N6 has gradually replaced $\mathrm{H} 5 \mathrm{~N} 1$ as a dominant subtype in poultry, especially in Southern China (Bi et al., 2016b). H5N6 has caused severe outbreaks amongst poultry in Southeast Asia and has also been found in wild birds in some regions of Asia and even in Europe after 2014 (Bi et al., 2016c) (http://www. oie.int/en/animal-health-in-the-world/update-on-avian-influenza). There is a real risk that H5N6 may also transmit globally, following the footsteps of H5N1 and H5N8.

\section{THE MOLECULAR MECHANISM OF CROSS- SPECIES AIV INFECTION}

There are at least two host barriers for AIVs to cross-infect mammalian cells. The first barrier is receptor binding, in which AIVs require the human-type ( $\alpha 2-6-\mathrm{SA})$ receptor binding ability to infect human cells. The second is that the viral ribonucleoprotein (vRNP) complex-polymerase of AIVs should function well in the new host cells for efficient virus replication. Our studies on the first barrier showed at the atomic level that the molecular mechanism of transmissibility of H5N1 viruses among ferrets caused by seven critical mutations in the HA protein (Lu et al., 2013; Zhang et al., 2013a). H1N1 viruses with the D225G mutation was found to have developed an ability to bind both human-type $(\alpha 2-6-\mathrm{SA})$ and avian-type ( $\alpha 2-3-\mathrm{SA})$ receptors (Zhang et al., 2013b), thus elucidating the reason for severe lower respiratory disease in humans. Critical atoms associated to receptor binding were also identified in $\mathrm{H} 4, \mathrm{H} 6$ and $\mathrm{H} 10-$ subtype influenza $\mathrm{A}$, as well as influenza $\mathrm{D}$ viruses (Song et al., 2016; Song et al., 2017; Wang et al., 2015a; Wang et al., 2015b). We discovered that the novel H7N9 LPAIV with G226L mutation on HA possessed dual receptor binding properties (Shi et al., 2013), which explained why H7N9 was able to cross the first species barrier to infect humans. Stu- 
dies on the second barrier showed that the PB2 gene was critical for H7N9 virus replication in human cells with high RNP activities, and was identified as an important determinant of virulence in mice (Bi et al., 2015c). Other genes, including HA and NA, also contributed to the infectivity of H7N9 in human cells. NA with a five-amino-deletion in the stalk region could not influence the virulence of H7N9 in mice, but a longer deletion in the NA stalk increased the pathogenicity of H7N9 in mice (Bi et al., 2015b). Uncontrolled cytokine release were identified in the infected hosts (Bi et al., 2015c; Bi et al., 2016d), and considered as an underlying reason for the high mortality caused by LPAIV H7N9 infections.

Several novel nuclear export signals (NES) identified in the NP, M1 and NS2, as well as phosphorylation sites in M1 and NS1, may work syngergistically for the nuclear export of vRNA, which is crucial for influenza A virus replication (Cao et al., 2012; Gao et al., 2014; Li et al., 2017; Wang et al., 2013; Yu et al., 2012; Zheng et al., 2017). The NES and nuclear localization signal (NLS) were also identified in M1 of influenza B virus (Cao et al., 2014).

\section{HOST-VIRUS INTERACTION}

The interactions between host factors and the virus are crucial for viral infectivity and host responses. Host factors were investigated by CASCIRE for the pathogenesis of influenza virus. For example, Cyclophilin A and NEDD8 (neural precursor cell expressed developmentally down-regulated 8) inhibited virus replication through interactions with $\mathrm{M} 1$ and PB2 of influenza virus, respectively (Liu et al., 2009; Liu et al., 2012). Cyclophilin A was also identified as a regulator controlling the severity of disease development caused by an uncontrolled immune response after infection ( $\mathrm{Li}$ et al., 2016). Cyclin T1/CDK9 (cyclin-dependentkinases 9) was found to increase virus replication through up-regulating the transcription activity of vRNP (Zhang et al., 2010). microRNA-33a was found to disturb influenza A virus replication by targeting ARCN1 and inhibiting vRNP activity and virus replication (Hu et al., 2016).

Host factors involved with innate immunity during influenza virus infections were identified, such as Ndfip1, which was identified as an inhibitor of MAVS-mediated antiviral response (Wang et al., 2012). The antiviral effect of RIG-Imediated IFN response was found to be regulated by T80 phosphorylation of the NS1 protein in influenza A viruses (Zheng et al., 2017). Interestingly, while influenza A virus NS1 protein interacts with RIG-I and TRIM25 to suppress the activation of RIG-I-mediated signaling, influenza B virus NS1 protein was unable to directly interact with RIG-I, but instead engages in the formation of a RIG-I/TRIM25/NS1-B ternary complex (Jiang et al., 2016). The non-coding RNAs were also discovered to modulate the antiviral interferon response against influenza A virus (Ma et al., 2016; Ouyang et al., 2014).

\section{THE MOLECULAR MECHANISM OF DRUG RESISTANCE AND ANTIVIRAL MEASURES}

Due to a broad M2-mediated inhibitor resistance to influenza A viruses (e.g. 2009 pandemic H1N1 and H7N9), neuraminidase inhibitors (NAIs) currently constitute the dominant class of anti-influenza drugs in clinics. However, NAIs (e.g. zanamivir) are more effective against group 1 than group 2 influenza A viruses, because of differences in the NA molecular structures (Li et al., 2010; Vavricka et al., 2011). In addition, NAI resistant strains were gradually identified in clinics during the NAI treatments. To address this, we explored the effect of older, general antiviral drugs, such as ribavirin, which worked as well as zanamivir against the H7N9 infections in mice (Bi et al., 2016e). We also developed and tested new compounds against NAI-resistant viruses based on the molecular mechanism of NAI-resistance, such as tetravalent zanamivir that presented outstanding activities against $\mathrm{H} 7 \mathrm{~N} 9$ and $\mathrm{H} 3 \mathrm{~N} 2$ infections (Fu et al., 2016; Wu et al., 2013). Studies on vaccine (http://www. im.cas.cn/xwzx/jqyw/201705/t20170527_4805236.html) and human antibody development against influenza viruses, as well as the emerging and re-emerging pathogens risk to China (Dai et al., 2016; Wang et al., 2016; Wu et al., 2015), were also performed by CASCIRE.

The ability to provide early-warning for outbreaks, thus leading to the development of antiviral measures (drugs, vaccines and human antibodies) for influenza viruses and other novel pathogens are the aims alongside elucidation of pathogenesis mechanisms. For One Health, CASCIRE hopes to establish future collaborations with worldwide research groups, expand surveillance efforts and promote the earlywarning ability against emerging and re-remerging infectious diseases.

Compliance and ethics The author(s) declare that they have no conflict of interest.

Acknowledgements This work was supported by the National Key $R \& D$ Program of China (2016YFE0205800), National Science and Technology Major Project (2016ZX10004222), Emergency Technology Research Issue on Prevention and Control for Human Infection with A(H7N9) Avian Influenza Virus (10600100000015001206), intramural special grants for influenza virus research from the Chinese Academy of Sciences (KJZD-EWL15), Tianjin Research Program of the Application Foundation and Advanced Technology (14JCYBJC24400) and the research project RFBR 1704-01919. George F. Gao is a leading principal investigator of the NSFC Innovative Research Group (81621091). Yuhai Bi is supported by the Youth Innovation Promotion Association of Chinese Academy of Sciences (CAS) (2017122). 
Bi, Y., Mei, K., Shi, W., Liu, D., Yu, X., Gao, Z., Zhao, L., Gao, G.F., Chen, J., and Chen, Q. (2015a). Two novel reassortants of avian influenza A (H5N6) virus in China. J Gene Virol 96, 975-981.

Bi, Y., Wong, G., Liu, Y., Liu, L., Gao, G.F., and Shi, Y. (2016e). Ribavirin is effective against drug-resistant H7N9 influenza virus infections. Protein Cell 7, 611-614.

Bi, Y., Xiao, H., Chen, Q., Wu, Y., Fu, L., Quan, C., Wong, G., Liu, J., Haywood, J., Liu, Y., Zhou, B., Yan, J., Liu, W., and Gao, G.F. (2015b). Changes in the length of the neuraminidase stalk region impact H7N9 virulence in mice. J Virol 90, 2142-2149.

Bi, Y., Xie, Q., Zhang, S., Li, Y., Xiao, H., Jin, T., Zheng, W., Li, J., Jia, X., Sun, L., Liu, J., Qin, C., Gao, G.F., and Liu, W. (2015c). Assessment of the internal genes of influenza A (H7N9) virus contributing to high pathogenicity in mice. J Virol 89, 2-13.

Bi, Y., Zhang, Z., Liu, W., Yin, Y., Hong, J., Li, X., Wang, H., Wong, G., Chen, J., Li, Y., Ru, W., Gao, R., Liu, D., Liu, Y., Zhou, B., Gao, G.F., Shi, W., and Lei, F. (2015d). Highly pathogenic avian influenza A (H5N1) virus struck migratory birds in China in 2015. Sci Rep 5, 12986.

Bi, Y., Chen, J., Zhang, Z., Li, M., Cai, T., Sharshov, K., Susloparov, I., Shestopalov, A., Wong, G., He, Y., Xing, Z., Sun, J., Liu, D., Liu, Y., Liu, L., Liu, W., Lei, F., Shi, W., and Gao, G.F. (2016a). Highly pathogenic avian influenza H5N1 Clade 2.3.2.1c virus in migratory birds, 2014-2015. Virol Sin 31, 300-305.

Bi, Y., Chen, Q., Wang, Q., Chen, J., Jin, T., Wong, G., Quan, C., Liu, J., Wu, J., Yin, R., Zhao, L., Li, M., Ding, Z., Zou, R., Xu, W., Li, H., Wang, H., Tian, K., Fu, G., Huang, Y., Shestopalov, A., Li, S., Xu, B., Yu, H., Luo, T., Lu, L., Xu, X., Luo, Y., Liu, Y., Shi, W., Liu, D., and Gao, G.F. (2016b). Genesis, evolution and prevalence of H5N6 avian influenza viruses in China. Cell Host Microbe 20, 810-821.

Bi, Y., Liu, H., Xiong, C., Di Liu, C., Shi, W., Li, M., Liu, S., Chen, J., Chen, G., Li, Y., Yang, G., Lei, Y., Xiong, Y., Lei, F., Wang, H., Chen, Q., Chen, J., and Gao, G.F. (2016c). Novel avian influenza A (H5N6) viruses isolated in migratory waterfowl before the first human case reported in China, 2014. Sci Rep 6, 29888.

Bi, Y., Liu, J., Xiong, H., Zhang, Y., Liu, D., Liu, Y., Gao, G.F., and Wang, B. (2016d). A new reassortment of influenza A (H7N9) virus causing human infection in Beijing, 2014. Sci Rep 6, 26624.

Cao, S., Liu, X., Yu, M., Li, J., Jia, X., Bi, Y., Sun, L., Gao, G.F., and Liu, W. (2012). A nuclear export signal in the matrix protein of influenza A virus is required for efficient virus replication. J Virol 86, 4883-4891.

Cao, S., Jiang, J., Li, J., Li, Y., Yang, L., Wang, S., Yan, J., Gao, G.F., and Liu, W. (2014). Characterization of the nucleocytoplasmic shuttle of the matrix protein of influenza B virus. J Virol 88, 7464-7473.

Cui, L., Liu, D., Shi, W., Pan, J., Qi, X., Li, X., Guo, X., Zhou, M., Li, W., Li, J., Haywood, J., Xiao, H., Yu, X., Pu, X., Wu, Y., Yu, H., Zhao, K., Zhu, Y., Wu, B., Jin, T., Shi, Z., Tang, F., Zhu, F., Sun, Q., Wu, L., Yang, R., Yan, J., Lei, F., Zhu, B., Liu, W., Ma, J., Wang, H., and Gao, G.F. (2014). Dynamic reassortments and genetic heterogeneity of the human-infecting influenza A (H7N9) virus. Nat Commun 5, 3142.

Dai, L., Song, J., Lu, X., Deng, Y.Q., Musyoki, A.M., Cheng, H., Zhang, Y., Yuan, Y., Song, H., Haywood, J., Xiao, H., Yan, J., Shi, Y., Qin, C.F., Qi, J., and Gao, G.F. (2016). Structures of the Zika virus envelope protein and its complex with a flavivirus broadly protective antibody. Cell Host Microbe 19, 696-704.

Fu, L., Bi, Y., Wu, Y., Zhang, S., Qi, J., Li, Y., Lu, X., Zhang, Z., Lv, X., Yan, J., Gao, G.F., and Li, X. (2016). Structure-based tetravalent zanamivir with potent inhibitory activity against drug-resistant influenza viruses. J Med Chem 59, 6303-6312.

Gao, G.F. (2014). Influenza and the live poultry trade. Science 344, $235-$ 235.

Gao, S., Wang, S., Cao, S., Sun, L., Li, J., Bi, Y., Gao, G.F., and Liu, W. (2014). Characteristics of nucleocytoplasmic transport of H1N1 influenza A virus nuclear export protein. J Virol 88, 7455-7463.

Hu, X., Liu, D., Wang, M., Yang, L., Wang, M., Zhu, Q., Li, L., and Gao, G.F. (2011). Clade 2.3.2 avian influenza virus (H5N1), Qinghai Lake Region, China, 2009-2010. Emerg Infect Dis 17, 560-562.
Hu, Y., Jiang, L., Lai, W., Qin, Y., Zhang, T., Wang, S., and Ye, X. (2016). microRNA-33a disturbs influenza A virus replication by targeting ARCN1 and inhibiting viral ribonucleoprotein activity. J Gene Virol 97, $27-38$.

Jiang, J., Li, J., Fan, W., Zheng, W., Yu, M., Chen, C., Sun, L., Bi, Y., Ding, C., Gao, G.F., and Liu, W. (2016). Robust Lys63-linked ubiquitination of RIG-I promotes cytokine eruption in early influenza B virus infection. J Virol 90, 6263-6275.

Li, J., Chen, C., Wong, G., Dong, W., Zheng, W., Li, Y., Sun, L., Zhang, L., Gao, G.F., Bi, Y., and Liu, W. (2016). Cyclophilin A protects mice against infection by influenza A virus. Sci Rep 6, 28978.

Li, J., Zheng, W., Hou, L., Chen, C., Fan, W., Qu, H., Jiang, J., Liu, J., Gao, G.F., Zhou, J., Sun, L., and Liu, W. (2017). Differential nucleocytoplasmic shuttling of the nucleoprotein of influenza a viruses and association with host tropism. Cell Microbiol 19, e12692.

Li, Q., Qi, J., Zhang, W., Vavricka, C.J., Shi, Y., Wei, J., Feng, E., Shen, J., Chen, J., Liu, D., He, J., Yan, J., Liu, H., Jiang, H., Teng, M., Li, X., and Gao, G.F. (2010). The 2009 pandemic H1N1 neuraminidase N1 lacks the 150-cavity in its active site. Nat Struct Mol Biol 17, 1266-1268.

Liu, D., Shi, W., Shi, Y., Wang, D., Xiao, H., Li, W., Bi, Y., Wu, Y., Li, X., Yan, J., Liu, W., Zhao, G., Yang, W., Wang, Y., Ma, J., Shu, Y., Lei, F., and Gao, G.F. (2013). Origin and diversity of novel avian influenza A H7N9 viruses causing human infection: phylogenetic, structural, and coalescent analyses. Lancet 381, 1926-1932.

Liu, D., Shi, W., and Gao, G.F. (2014). Poultry carrying H9N2 act as incubators for novel human avian influenza viruses. Lancet 383, 869.

Liu, J., Xiao, H., Lei, F., Zhu, Q., Qin, K., Zhang, X.W., Zhang, X.L., Zhao, D., Wang, G., Feng, Y., Ma, J., Liu, W., Wang, J., and Gao, G.F. (2005). Highly pathogenic $\mathrm{H} 5 \mathrm{~N} 1$ influenza virus infection in migratory birds. Science 309, 1206-1206.

Liu, X., Sun, L., Yu, M., Wang, Z., Xu, C., Xue, Q., Zhang, K., Ye, X., Kitamura, Y., and Liu, W. (2009). Cyclophilin A interacts with influenza A virus M1 protein and impairs the early stage of the viral replication. Cell Microbiol 11, 730-741.

Liu, X., Zhao, Z., Xu, C., Sun, L., Chen, J., Zhang, L., and Liu, W. (2012). Cyclophilin A restricts influenza A virus replication through degradation of the M1 protein. PLoS ONE 7, e31063.

Lu, X., Shi, Y., Zhang, W., Zhang, Y., Qi, J., and Gao, G.F. (2013). Structure and receptor-binding properties of an airborne transmissible avian influenza A virus hemagglutinin H5 (VN1203mut). Protein Cell 4, 502511.

Ma, Y., Ouyang, J., Wei, J., Maarouf, M., and Chen, J.L. (2016). Involvement of host non-coding RNAs in the pathogenesis of the influenza virus. Int J Mol Sci 18, 39.

Ouyang, J., Zhu, X., Chen, Y., Wei, H., Chen, Q., Chi, X., Qi, B., Zhang, L., Zhao, Y., Gao, G.F., Wang, G., and Chen, J.L. (2014). NRAV, a long noncoding RNA, modulates antiviral responses through suppression of interferon-stimulated gene transcription. Cell Host Microbe 16, 616626.

Qi, W., Jia, W., Liu, D., Li, J., Bi, Y., Xie, S., Li, B., Hu, T., Du, Y., Xing, L., Zhang, J., Zhang, F., Wei, X., Eden, J.S., Li, H., Tian, H., Li, W., Su, G., Lao, G., Xu, C., Xu, B., Liu, W., Zhang, G., Ren, T., Holmes, E.C., Cui, J., Shi, W., Gao, G.F., and Liao, M. (2017). Emergence and adaptation of a novel highly pathogenic H7N9 influenza virus in birds and humans from a 2013-human-infecting low pathogenic ancestor. J Virol in press doi: 10.1128/JVI.00921-17.

Shi, Y., Zhang, W., Wang, F., Qi, J., Wu, Y., Song, H., Gao, F., Bi, Y., Zhang, Y., Fan, Z., Qin, C., Sun, H., Liu, J., Haywood, J., Liu, W., Gong, W., Wang, D., Shu, Y., Wang, Y., Yan, J., and Gao, G.F. (2013). Structures and receptor binding of hemagglutinins from human-infecting H7N9 influenza viruses. Science 342, 243-247.

Song, H., Qi, J., Khedri, Z., Diaz, S., Yu, H., Chen, X., Varki, A., Shi, Y., and Gao, G.F. (2016). An open receptor-binding cavity of hemagglutinin-esterase-fusion glycoprotein from newly-identified influenza D virus: basis for its broad cell tropism. PLoS Pathog 12, e1005411.

Song, H., Qi, J., Xiao, H., Bi, Y., Zhang, W., Xu, Y., Wang, F., Shi, Y., and Gao, G.F. (2017). Avian-to-human receptor-binding adaptation by inf- 
luenza A virus hemagglutinin H4. Cell Rep 20, 1201-1214.

Su, S., Bi, Y., Wong, G., Gray, G.C., Gao, G.F., and Li, S. (2015). Epidemiology, evolution, and recent outbreaks of avian influenza virus in China. J Virol 89, 8671-8676.

Vavricka, C.J., Li, Q., Wu, Y., Qi, J., Wang, M., Liu, Y., Gao, F., Liu, J., Feng, E., He, J., Wang, J., Liu, H., Jiang, H., and Gao, G.F. (2011). Structural and functional analysis of laninamivir and its octanoate prodrug reveals group specific mechanisms for influenza NA inhibition. PLoS Pathog 7, e1002249.

Wang, F., Qi, J., Bi, Y., Zhang, W., Wang, M., Zhang, B., Wang, M., Liu, J., Yan, J., Shi, Y., and Gao, G.F. (2015a). Adaptation of avian influenza A (H6N1) virus from avian to human receptor-binding preference. EMBO J 34, 1661-1673.

Wang, G., Zhan, D., Li, L., Lei, F., Liu, B., Liu, D., Xiao, H., Feng, Y., Li, J., Yang, B., Yin, Z., Song, X., Zhu, X., Cong, Y., Pu, J., Wang, J., Liu, J., Gao, G.F., and Zhu, Q. (2008). H5N1 avian influenza re-emergence of Lake Qinghai: phylogenetic and antigenic analyses of the newly isolated viruses and roles of migratory birds in virus circulation. J Gene Virol 89, 697-702.

Wang, M., Zhang, W., Qi, J., Wang, F., Zhou, J., Bi, Y., Wu, Y., Sun, H., Liu, J., Huang, C., Li, X., Yan, J., Shu, Y., Shi, Y., and Gao, G.F. (2015b). Structural basis for preferential avian receptor binding by the human-infecting H10N8 avian influenza virus. Nat Commun 6, 5600.

Wang, Q., Yang, H., Liu, X., Dai, L., Ma, T., Qi, J., Wong, G., Peng, R., Liu, S., Li, J., Li, S., Song, J., Liu, J., He, J., Yuan, H., Xiong, Y., Liao, Y., Li, J., Yang, J., Tong, Z., Griffin, B.D., Bi, Y., Liang, M., Xu, X., Qin, C., Cheng, G., Zhang, X., Wang, P., Qiu, X., Kobinger, G., Shi, Y., Yan, J., and Gao, G.F. (2016). Molecular determinants of human neutralizing antibodies isolated from a patient infected with Zika virus. Sci Transl Med 8, 369ra179.

Wang, S., Zhao, Z., Bi, Y., Sun, L., Liu, X., and Liu, W. (2013). Tyrosine 132 phosphorylation of influenza A virus M1 protein is crucial for virus replication by controlling the nuclear import of M1. J Virol 87, 61826191.

Wang, Y., Tong, X., and Ye, X. (2012). Ndfip1 negatively regulates RIG-Idependent immune signaling by enhancing E3 ligase Smurf1-mediated MAVS degradation. J Immunol 189, 5304-5313.

Wu, Y., Bi, Y., Vavricka, C.J., Sun, X., Zhang, Y., Gao, F., Zhao, M., Xiao,
H., Qin, C., He, J., Liu, W., Yan, J., Qi, J., and Gao, G.F. (2013). Characterization of two distinct neuraminidases from avian-origin human-infecting H7N9 influenza viruses. Cell Res 23, 1347-1355.

Wu, Y., Cho, M.S., Shore, D., Song, M., Choi, J.A., Jiang, T., Deng, Y.Q., Bourgeois, M., Almli, L., Yang, H., Chen, L.M., Shi, Y., Qi, J., Li, A., Yi, K.S., Chang, M.S., Bae, J.S., Lee, H.J., Shin, J.Y., Stevens, J., Hong, S.S., Qin, C.F., Gao, G.F., Chang, S.J., and Donis, R.O. (2015). A potent broad-spectrum protective human monoclonal antibody crosslinking two haemagglutinin monomers of influenza A virus. Nat Commun 6, 7708 .

Yu, M., Liu, X., Cao, S., Zhao, Z., Zhang, K., Xie, Q., Chen, C., Gao, S., Bi, Y., Sun, L., Ye, X., Gao, G.F., and Liu, W. (2012). Identification and characterization of three novel nuclear export signals in the influenza $\mathrm{A}$ virus nucleoprotein. J Virol 86, 4970-4980.

Zhang, F., Bi, Y., Wang, J., Wong, G., Shi, W., Hu, F., Yang, Y., Yang, L., Deng, X., Jiang, S., He, X., Liu, Y., Yin, C., Zhong, N., and Gao, G.F. (2017). Human infections with recently-emerging highly pathogenic H7N9 avian influenza virus in China. J Infect 75, 71-75.

Zhang, J., Li, G., and Ye, X. (2010). Cyclin T1/CDK9 interacts with influenza A virus polymerase and facilitates its association with cellular rna polymerase II. J Virol 84, 12619-12627.

Zhang, T., Bi, Y., Tian, H., Li, X., Liu, D., Wu, Y., Jin, T., Wang, Y., Chen, Q., Chen, Z., Chang, J., Gao, G.F., and Xu, B. (2014). Human infection with influenza virus $\mathrm{A}(\mathrm{H} 10 \mathrm{~N} 8)$ from live poultry markets, China, 2014. Emerg Infect Dis in press doi: 10.3201/eid2012.140911.

Zhang, W., Shi, Y., Lu, X., Shu, Y., Qi, J., and Gao, G.F. (2013a). An airborne transmissible avian influenza $\mathrm{H} 5$ hemagglutinin seen at the atomic level. Science 340, 1463-1467.

Zhang, W., Shi, Y., Qi, J., Gao, F., Li, Q., Fan, Z., Yan, J., and Gao, G.F. (2013b). Molecular basis of the receptor binding specificity switch of the hemagglutinins from both the 1918 and 2009 pandemic influenza A viruses by a D225G substitution. J Virol 87, 5949-5958.

Zheng, W., Cao, S., Chen, C., Li, J., Zhang, S., Jiang, J., Niu, Y., Fan, W., Li, Y., Bi, Y., Gao, G.F., Sun, L., and Liu, W. (2017). Threonine 80 phosphorylation of non-structural protein 1 regulates the replication of influenza A virus by reducing the binding affinity with RIG-I. Cell Microbiol 19, e12643. 